\title{
El papel de la segunda persona en la constitución del autoconocimiento*
}

\author{
The role of the second person in the constitution \\ of self-knowledge
}

\begin{abstract}
Resumen: En este trabajo defenderé la existencia, en el desarrollo ontogenético, tanto de una perspectiva como de una autoridad de segunda persona en la atribución mental. La perspectiva de segunda persona está en la base de la capacidad del bebé de descubrirse como agente, origen de la perspectiva de primera persona, pero también como objeto de atención, origen de la perspectiva de tercera persona. Defenderé, asimismo, que es mediante la autoridad de segunda persona por la que el bebé aprende a normativizar su conducta, tanto ocasionalmente como a largo plazo. Finalmente, discutiré brevemente la posibilidad de que la influencia de esta perspectiva continúe a lo largo de la vida del sujeto.

Palabras clave: Segunda persona, atención conjunta, triangulación, perspectiva, autoridad epistémica, autoconocimiento.
\end{abstract}

JOSÉ FERRER DE LUNA**

En este trabajo esbozaré la idea de que existe, en el desarrollo ontogenético, tanto una perspectiva como una autoridad de segunda persona en la atribución mental. La perspectiva de segunda persona es condición de posibilidad de la capacidad del bebé de

Fecha de recepción: 19/12/2012. Fecha de aceptación final: 21/05/2013.

* Este artículo se ha beneficiado enormemente de los comentarios y sugerencias de Neftalí Villanueva, Nemesio García-Carril, Pablo Fernández Rojas, Víctor Fernández, Manuel Heras, y de dos evaluadores anónimos de Daimon. Ha sido elaborado dentro del Programa de Becas de Iniciación del Plan Propio de Investigación de la Universidad de Granada (convocatoria 2011), bajo la supervisión de Manuel de Pinedo, a quien debo un agradecimiento especial por el gran apoyo brindado tanto en el ámbito académico, como en el personal.

** Becario de Colaboración del Ministerio de Educación, Cultura y Deporte, vinculado al proyecto de investigación «Holismo, disposiciones y agencia» (Ministerio de Educación y Ciencia, FFI2010-19455). En la actualidad cursa el Máster en Filosofía Contemporánea de la Universidad de Granada. Correo electrónico: jfdluna@ correo.ugr.es. Su línea de investigación es la epistemología y el autoconocimiento. 
descubrirse como agente, origen de la perspectiva de primera persona, pero también como objeto de atención, origen de la perspectiva de tercera persona. Defenderé, asimismo, que es mediante la autoridad de segunda persona por la que el bebé aprende a normativizar su conducta, tanto ocasionalmente como a largo plazo. Finalmente, discutiré brevemente la posibilidad de que la influencia de esta perspectiva continúe a lo largo de la vida del sujeto.

\section{Descartes y Ryle: dos posturas extremas}

Los dos paradigmas históricos más reconocibles respecto al autoconocimiento están representados en los trabajos de Descartes (1641) y Ryle (1949). Éstos defienden, respectivamente, que el acceso a nuestros estados mentales ocurre exclusivamente desde una perspectiva de primera y tercera persona. Estamos, pues, ante dos puntos de vista extremos. Bajo el esquema cartesiano se entiende que el autoconocimiento deriva de un sentido interno por el cual tenemos un acceso directo, completo e infalible a nuestros estados mentales. Desde este punto de vista, la perspectiva subjetiva, de primera persona, es la única mediante la cual podemos conocernos, es decir, es la única mediante la cual podemos tener dicho acceso sin riesgo de error. De otra parte, para Ryle, en el acceso de tercera persona el autoconocimiento deriva de una serie de auto-adscripciones de los estados mentales hechas a partir de evidencia externa, inferencias, análisis o autointerpretaciones. Desde esta perspectiva, la observación del comportamiento, tanto lingüístico como no lingüístico, es la principal base sobre la que se obtiene el acceso a nuestros estados mentales.

La primera postura invalida la perspectiva de tercera persona con respecto al propio pensamiento y la segunda hace desaparecer la perspectiva de primera persona. Descartes anula la perspectiva de tercera persona con su dualismo epistemológico, en el cual afirma que el único conocimiento indubitable que podemos tener es el que se da mediante el acceso privilegiado que tenemos en primera persona a través de la introspección, mientras que Ryle niega la existencia de un acceso privilegiado y, con ello, la existencia de una perspectiva de primera persona, ya que, como hemos visto, para él no existe una diferencia, sino de grado, entre la perspectiva de tercera y la de primera persona, reduciendo, de esta manera, la segunda a la primera.

Pero estas son únicamente diferencias de grado, no de clase. La superioridad que posee el que habla del conocimiento de lo que está haciendo, sobre el conocimiento que tiene el que escucha, no indica que aquél posea un Acceso Privilegiado a hechos de un tipo inevitablemente inaccesible para éste, sino, solamente, que está en mejor posición para conocerlos. [...] Pero en ninguno de los sentidos en que comúnmente apreciamos si una persona sabe, o no, algo acerca de sí misma, es necesario o útil postular un Acceso Privilegiado para explicar cómo es que ha obtenido o podría haber obtenido tal conocimiento. (Ryle 1949, 203s)

Ryle niega el cartesianismo pero de otra parte le concede demasiado. Niega que exista un acceso privilegiado, pero le concede que, de existir, éste debe ser infalible. Así llega a la conclusión de que como dicha infalibilidad no existe, tampoco existe dicho acceso pri- 
vilegiado. Ryle cae, pues, en el error de asumir la infalibilidad como condición necesaria del acceso en primera persona. Sin embargo, ésta no es la única forma de concebirlo, como veremos más adelante ${ }^{1}$.

\section{Davidson: la autoridad de primera persona, la triangulación necesaria para el cono- cimiento objetivo y su extensión al autoconocimiento}

En «La autoridad de primera persona», D. Davidson (1984) señala que Ryle rechaza la asimetría entre las perspectivas de primera y de tercera persona sin dar una explicación de ello.

Ryle ni acepta ni explica la asimetría entre las afirmaciones en primera persona del presente acerca de actitudes y las afirmaciones de otras personas y otros tiempos; simplemente niega que exista. (Davidson 1984, 29)

En este ensayo, Davidson da una explicación de la autoridad de primera persona basándose en sus ideas con respecto a la interpretación radical. Sin embargo, hay material en otros trabajos de Davidson para explicar el carácter privilegiado, pero no infalible, de la perspectiva de primera persona dando cuenta al mismo tiempo de las condiciones de posibilidad de la emergencia del pensamiento y el autoconocimiento. En «Animales racionales» y en «Tres variedades de conocimiento» Davidson puso de manifiesto la necesidad de la triangulación entre dos perspectivas y un tercer elemento externo al cual los dos primeros refieren, para que emerja el conocimiento objetivo.

En «Animales racionales», Davidson (1982) apunta a una triangulación entre el hablante, el oyente y un mundo exterior por ambos compartido, triangulación que sólo es posible mediante la comunicación que se da a través del lenguaje.

Nuestro sentido de la objetividad es consecuencia de otra clase de triangulación, una que requiere dos criaturas. Cada una de ellas interacciona con un objeto, pero lo que da a cada una el concepto de cómo son las cosas objetivamente es la línea de base que se forma entre las criaturas mediante el lenguaje. Sólo el hecho de que compartan un concepto de verdad da sentido a la afirmación de que tienen creencias, de que son capaces de asignar un lugar a los objetos en el mundo público². (Davidson 1982,154 s)

De esta manera, la existencia de un mundo externo, de una criatura semejante y de un lenguaje son, según Davidson, condiciones necesarias y conjuntamente suficientes para tener

1 Una maniobra similar está detrás del eliminativismo con respecto a la mente defendido por Rorty en los años 60. Su argumento tiene, como el de Ryle, la estructura de un modus tollens: la característica central de la mente es el acceso incorregible a sus propios contenidos; pero ningún acceso es incorregible, luego la mente no existe. Ver Rorty (1965) y Rorty (1970).

2 La noción de objetividad con la que trabajaré a lo largo de este trabajo es la que se sigue de la explicación davidsoniana de la emergencia del pensamiento objetivo como consecuencia de la triangulación. Algo es objetivo, por tanto, si puede ser el resultado de la comparación intersubjetiva de puntos de vista. 
conocimiento objetivo. Esta es la idea que amplía y argumenta en su ensayo «Tres variedades de conocimiento» (Davidson 1991). En él sostiene la irreductibilidad del conocimiento a ninguna de las tres maneras que tenemos de conocer, a saber, al conocimiento de mi propia mente (lo que pensamos, deseamos, creemos...), al conocimiento del mundo externo (de los objetos que captamos a través de los sentidos) o al conocimiento de las otras mentes (lo que los otros creen, desean, tienen intención de hacer...). A pesar de los intentos de los enfoques reduccionistas de restringir el conocimiento a una o dos de sus variedades, los problemas epistemológicos que conlleva, es decir, el problema del conocimiento del mundo externo, el del conocimiento de otras mentes y el del conocimiento no mediado de nuestras propias mentes, ni pueden reducirse a uno o dos de ellos ni deben, por tanto, tratarse por separado. Cada una de las tres variedades de conocimiento depende de las otras dos. Según Davidson, nuestros pensamientos poseen contenido proposicional como consecuencia de formar parte de una comunidad de comunicación, pues sólo la comunicación interpersonal permite que los hablantes reconozcamos la pertenencia a un mundo compartido. Sin la intercomunicación mis respuestas a los objetos no podrían llamarse lenguaje y mi ubicación de los estímulos que percibo no podría tener ninguna objetividad. Es necesario que el hablante y el oyente compartan reacciones a estímulos comunes, pues «sin este compartir el pensamiento y el habla no tendrían ningún contenido en particular [...]. Son necesarios dos puntos de vista para asignar una ubicación a la causa de un pensamiento y para, de ese modo, definir su contenido» (Davidson 1991, 290). El conocimiento de otras mentes y el conocimiento del mundo dependen el uno del otro puesto que ninguno es posible sin el otro, sin el conocimiento de otra mente no es posible el conocimiento del mundo pues, como ya hemos dicho, «la clase de triangulación que es esencial para el pensamiento requiere que los que se comunican reconozcan que ocupan posiciones en un mundo compartido» (Davidson 1991, 291). Asimismo, el conocimiento de nuestra propia mente y el conocimiento de la mente de los demás dependen el uno del otro puesto que no podemos atribuir pensamientos a los demás a menos que los tengamos nosotros mismos, es decir, a menos que sepamos lo que pensamos. ${ }^{3}$

Cristina Borgoni (2009, 253ss) ha desarrollado algunas de las ideas de Davidson aplicándolas al autoconocimiento, extendiendo la necesidad de la triangulación para el conocimiento objetivo a la necesidad de una triangulación entre la perspectiva de primera persona, la de tercera persona y los estados mentales. Para Borgoni, hay autoridad de primera persona porque hay triangulación entre ambas perspectivas y los estados mentales: «Argumento que la autoridad es más bien un atributo legítimo de una persona en tanto que ella es la única capaz de tener perspectiva de primera y de tercera persona sobre sí misma.» (Borgoni 2009, 253). Borgoni se basa parcialmente en la idea de Bensusan y Pinedo (2007) según la cual la mejor forma de entender la exigencia de Richard Moran (2001), de que el carácter normativo del auto-conocimiento es consecuencia de la capacidad del sujeto de acceder a su propio pensamiento por medio de una perspectiva de primera y otra de tercera persona, es en términos de la imagen davidsoniana de la triangulación.

3 Atribuir pensamientos a los demás implica el Principio de Correspondencia, es decir, considerar que el hablante responde a los mismos rasgos del mundo a los que uno respondería en similares circunstancias. Este principio y el Principio de Coherencia, el atribuir cierto grado de consistencia lógica en el pensamiento del hablante, son lo que Davidson denomina 'principios de caridad', principios con los que hemos de contar si queremos que sea posible la comunicación. 
Bajo este modelo, que suscribo, para poder hablar de autoconocimiento debemos tener en cuenta la necesidad de que el sujeto posea dos tipos de perspectivas sobre sus pensamientos y estados mentales. De esta manera tenemos que uno, por sí mismo con el acceso de primera persona, no es plenamente competente para el conocimiento de sus propios estados mentales, es decir, para el autoconocimiento, pues siempre está sujeto a condiciones de corrección que llegan desde la posibilidad de contraste entre ambas perspectivas. Supongamos, por ejemplo, que nos encontramos en una sala llena de gente y nos dirigimos a nuestra pareja para decirle «estoy muy irritado con el ruido que arma esta gente», a lo que ella nos contesta corrigiéndonos «no, a ti lo que te pasa es que tienes hambre». Podemos de inmediato darnos cuenta de que realmente tenemos hambre y que tal vez sea ese el motivo y no el ruido el que nos hace estar irritados (suponiendo que seamos alguien que se irrita cuando está hambriento). Únicamente mediante nuestra introspección no habríamos sido capaces de darnos cuenta de que el motivo de nuestra irritación no era el ruido del gentío sino, más bien, nuestra necesidad de comer, la cual estaba insatisfecha. Hemos, pues, aplicado ambas perspectivas a nosotros mismos, la nuestra propia, de primera persona, y otra observacional, la de alguien ajeno a nosotros, nuestra pareja en este caso, de tercera persona. Aun cuando pudiéramos considerar la perspectiva de tercera persona como un tránsito a una adscripción en primera persona, la perspectiva de tercera parece tener un papel indispensable para llegar a ser objetivos con nosotros mismos, pues es mediante el contraste entre ambas perspectivas por lo que podemos obtener objetividad sobre nuestros propios estados mentales ${ }^{4}$.

\section{El descubrimiento del niño como agente a través de la perspectiva de segunda per- sona. La atención conjunta}

Habiendo descartado las posturas exclusivas de Descartes y de Ryle, basándome en la triangulación davidsoniana de la objetividad del conocimiento y en la extensión de Borgoni de dicha triangulación al autoconocimiento, he expuesto la necesidad de poseer tanto una perspectiva de primera persona como una de tercera hacia nuestros propios estados mentales para dar cuenta del autoconocimiento. Pero, ¿cómo es esto posible?, ¿cómo puede uno realizar autoatribuciones desde ambas perspectivas? Para responder a esta pregunta debemos retrotraernos al desarrollo ontogenético y a la iniciación en las prácticas normativas.

Cuando nacemos no somos conscientes de nosotros mismos. Los teóricos del desarrollo ontogenético consideran que la atención conjunta cumple un papel indispensable en la aparición del pensamiento y la adquisición del lenguaje. Los fenómenos de atención conjunta están relacionados con el establecimiento de la interrelación que se da entre dos sujetos cuando prestan atención al mismo rasgo del entorno y, en especial, al surgimiento primigenio de este tipo de percepción recíproca del otro y de sus reacciones ante el mundo.

4 Hay muchas otras situaciones en las que se puede observar la importancia de la perspectiva de tercera persona. Otro ejemplo podría ser aquel en el que una persona cree y afirma no ser nacionalista, sabiendo lo que significa ser nacionalista, hasta que alguien le señala que aun cuando crea que no lo es, su comportamiento demuestra lo contrario, ya que vive apasionadamente la victoria del equipo de fútbol de su nación, por ejemplo, o ha votado en contra de la inserción de los inmigrantes. Estos ejemplos han sido tomados de Borgoni (2009). 
Hay un gran número de pruebas experimentales que se han venido realizando desde finales de los años 60 que muestran como incluso los bebés, a muy temprana edad, se implican en formas de 'relación interpersonal' que divergen sustancialmente del modo en que se relacionan con los objetos inanimados. Ya en las dos primeras semanas de vida, la voz humana interrumpe de una manera más efectiva el llanto infantil que un sonido artificial o que el típico sonajero. Antes de llegar a los dos meses, los bebés detienen el llanto y muestran un interés alerta y continuado cuando una persona entra en su campo visual y comienzan a llorar cuando desaparece, aun cuando antes estuvieran contentos (Wolff 1969, c.p. Hobson 1993, 53). Un estudio realizado por Brazelton, Koslowski y Main (Brazelton et al. 1974, c.p. Hobson 1993, 53) en torno a las diferentes respuestas que los bebés de cuatro semanas mantienen ante un objeto situado a su alcance, frente a ellos, por una parte, y ante la interacción cara a cara con su madre (o la persona que lo cuida) ${ }^{5}$, por otra, suscriben esta misma idea:

Era tanta la diferencia entre la atención, las vocalizaciones, las sonrisas y las conductas motoras con el objeto inanimado en comparación con las producidas ante la madre que teníamos la sensación de que nos bastaba con mirar a cualquier segmento del cuerpo del niño para detectar si estaba mirando al objeto o interactuando con su madre. (Branzenton et al. 1974, 53, c.p. Hobson 1993, 53)

Asimismo, diversos estudios de psicólogos evolutivos muestran la existencia de patrones de sintonización ajustada en los intercambios interpersonales cara a cara que se dan entre el bebé y su madre en los primeros meses de vida. Así, por ejemplo, Jeffey Cohn y Edward Tronick (Cohn y Tronick 1983, c.p. Hobson 1993, 54) llevaron a cabo un estudio en el que se pedía a las madres que mantuviesen un gesto deprimido durante un periodo de tres minutos de interacción cara a cara con el bebé de tres meses de edad. Los resultados mostraron que el bebé se mostraba cada vez más negativo llegando incluso a disgustarse y protestar. Otro estudio llevado a cabo por Jeanette Haviland y Mary Lelwica (Haviland y Lelwika 1987, c.p. Hobson 1993, 53). con bebés de 10 semanas de vida, revela los diferentes estados afectivos que manifiestan los bebés en relación a los gestos emocionales de sus madres en los intercambios cara a cara con éstas. Así, por ejemplo, frente a la expresión de alegría de la madre, los bebés respondían incrementando sus propias manifestaciones de alegría e interés, acompañadas de una mengua de las respuestas bucales, mientras que estas últimas aumentaban cuando la madre mostraba una expresión de tristeza. Asimismo, cuando la madre manifestaba una expresión de enfado, los bebés reducían los movimientos y mostraban un incremento de la ira.

Las configuraciones interpersonales de miradas mutuas y de intercambios faciales, vocales y gestuales no sólo parecen implicar una coordinación de las conductas de los bebés y las madres, sino también alguna clase de vínculo psocológico que cuando

5 Utilizo la relación del niño con su madre pues el caso más común. En adelante, cuando use el término 'madre', en este contexto, ha de entenderse como referido a la persona que está al cuidado del bebé, sea ésta la madre o no. 
se establece - $\mathrm{o}$ cuando se rompe - tiene consecuencias psicológicas para ambos participantes. En un cierto sentido, el bebé (o algún «mecanismo» dentro de él) parece esperar las formas adecuadas de respuestas expresivas, corporales y dinámicas, por parte de la otra persona. (Hobson 1993, 55) ${ }^{6}$

Aun cuando el contacto atento entre el niño y el adulto se da ya desde muy temprano, especialmente el contacto cara a cara centrado en la mirada, es hacia el primer año de vida (Hobson 1993, 55), después de los nueve meses, cuando podemos ver al bebé y al que lo cuida atendiendo juntos y de un modo duradero a rasgos del entorno. A partir de los ocho meses, aproximadamente, el bebé es capaz de coordinar acciones y actitudes interpersonales con su madre. En los tres meses siguientes, el bebé adquiere una serie de capacidades nuevas como son, entre otras, el seguimiento de la mirada de la otra persona, la determinación del punto a la que ésta señala, o la de indicar o mostrar objetos a otros (a menudo mirando a los ojos de la otra persona para comprobar si está atendiendo). (Bretherton et al. 1981, c.p. Hobson 1993, 56). Es entonces cuando podemos afirmar que la atención conjunta comienza a manifestarse. Este triángulo que forma la atención conjunta (bebé-adulto-entorno) no es un mero contacto de atención casual o esporádica, sino algo relativamente duradero en el que la actividad del niño y la del adulto se estructura en función de ese estado de atención compartido por ellos y hacia el entorno.

A pesar de no contar con una definición precisa de la atención conjunta, Naomi Elian ha ofrecido una definición que podríamos considerar como la más completa:

Del modo en que entiendo el término 'atención conjunta', decir de un evento que es un evento de dos (o más) sujetos que atienden conjuntamente al mismo objeto, supone estar comprometido, al menos, con la verdad de las siguientes cuatro afirmaciones:

a. Hay un objeto al cual cada sujeto atiende, que implica (i) una conexión causal entre el objeto y cada sujeto y (ii) conciencia del objeto por cada sujeto.

b. Existe una conexión causal, de algún tipo, entre los actos de los sujetos al atender al objeto.

c. Las experiencias de los dos sujetos hacen uso de su comprensión del concepto de atención.

d. Cada sujeto es consciente, en algún sentido, del objeto en tanto que objeto que está presente para ambos sujetos. Se da, en este sentido, una 'confluencia de mentes' entre ambos sujetos, de tal modo que el hecho de que ambos estén prestando atención al mismo objeto es abierta o mutuamente manifiesto. (Eilan 2005, 5)

Aun siendo la más completa de las definiciones dadas hasta el momento, la definición de Elian no es compartida por todos los teóricos de la atención conjunta. Las divergencias se encuentran en el grado en el que podemos atribuírselos a un niño de entre uno y dos años. El problema de fondo radica en indagar, teniendo en cuenta que sólo uno de los dos sujetos de la interacción es una criatura conceptual (el adulto), cómo el otro (el bebé) es

6 Este «mecanismo» que el bebé posee internamente está relacionado con la «lectura de atención» de la que hablaré más adelante. 
capaz llegar a constituir sus propios conceptos. Este espacio entre una criatura no conceptual y una criatura conceptual es lo que se ha denominado 'brecha ontogenética'. En su intento de superar dicha brecha, Ingar Brinck sostiene que «son las respuestas intencionales que responden a algún rasgo particular del contexto, las orientadas a un fin, y no las respuestas como meros comportamientos, las que preceden al estado conceptual» (Brinck 2004, 190).

El problema ahora es explicar en virtud de qué tipo de percepción la criatura es capaz de captar la intencionalidad teleológica de la acción sin recurrir a conceptos. La solución de Brinck pasa por distinguir dos tipos de mecanismos de discriminación, a saber, la discriminación lingüística, que emplea conceptos vinculados a los lenguajes naturales y la discriminación perceptiva, la cual es sensible al contexto y acondicionada al sujeto, dependiendo de las propiedades innatas de los sujetos y desarrollándose en la interacción con el entorno (Brinck 2004, 187). Para Brinck, por tanto, existe, a través de la discriminación perceptiva, un tipo de interacción en la que la comunicación intencional no tiene por qué ser lingüística. Así, afirma que «la atención conjunta constituye una manera de romper el círculo intencional precisamente porque es intencional sin ser proposicional» (Brinck 2004, 199). Esto es así porque aún sin que se detalle el contenido intencional puede haber atención conjunta, ya que percibir la atención no es sino percibir un estado que apunta a algo diferente del que atiende, sin importar, en primera instancia, el contenido. Es el fenómeno en sí de la atención y no el contenido intencional lo que el niño capta primeramente. La manera en la que el niño sabe cuál es el objeto de atención, es decir, el contenido, es mediante el seguimiento de la mirada del adulto hacia el objeto en cuestión. Esta percepción del estado de atención del adulto implica una capacidad primitiva del niño para el reconocimiento de intenciones denominada 'lectura de atención', la cual surge a partir de los nueve meses, siendo así que a partir del primer año, aproximadamente, cualquier niño es capaz de involucrarse en triángulos de atención conjunta.

Brink da una descripción de la lectura de atención en la que pone de manifiesto su anterioridad y necesidad para los triángulos de atención conjunta:

La lectura de atención ocurre cuando el oyente reconoce que el comportamiento de atención del hablante expresa un tipo particular de intención orientada a un objetivo y dirigida hacia un objeto en el espacio común. Dicha lectura se ejerce sobre la base de evidencia observacional y conductual obtenida de, por ejemplo, la expresión facial, la mirada y la postura corporal del hablante. La lectura de atención constituye el primer paso del proceso que conducirá a la atención conjunta. Al leer la atención de la hablante y estar interesado en ella, el oyente puede unirse a la hablante en este proceso. (Brinck 2004, 203)

El bebé, por tanto, tiene la predisposición a percibir la atención de su madre y esta predisposición ya supone un primer paso en la dirección de la captación de una perspectiva de segunda persona. En concreto, el objeto de atención de su madre que el bebé percibe primariamente es él mismo. Antes incluso de la aparición de la conciencia del 'tercer elemento' (un objeto externo al cual ambos dirigen la atención), en primera instancia, el bebé es consciente de la atención de su madre cuando se dirige hacia él mismo. Es este tipo de atención la que permitirá al bebé el descubrimiento de nuevos objetos de la atención de los 
demás. Vasudevi Reddy (Reddy 2008, 90ss) lleva a cabo un modelo del desarrollo de la conciencia de la atención desde una perspectiva de segunda persona. Para él, la conciencia de sí mismo como un objeto para los otros, parece no derivar de la posesión del concepto de sí mismo, sino de una sensación temprana de la atención y las actitudes de los otros dirigidas hacia uno mismo. Dicho de otra manera, es la perspectiva de segunda persona la que hace experimentar, mucho antes de la conceptualización, el 'sí mismo' como objeto para los demás (Reddy 2008, 121). Es a partir de esta experiencia, tras la conciencia de la atención hacía sí mismo, por la que el bebé llegará, gradualmente, a tomar conciencia de otros 'objetos' que ya no son él mismo y, por tanto, a participar en triángulos de atención conjunta. Un estudio realizado Farroni y sus colaboradores (Farroni et al. 2003, c.p. Reddy 2008), muestra que el seguimiento de la mirada del adulto por parte del bebé sólo ocurre si se ha establecido previamente la atención mutua, es decir, el bebé sólo sigue la mirada del adulto si éste ha fijado primeramente su mirada en él.

De esta forma, incluso antes de poseer ningún concepto (algo que solamente sucederá como consecuencia de su participación en ejercicios sofisticados de atención conjunta y de triangulación), el bebé ya capta, mediante la lectura de atención, la existencia de perspectivas, paradigmáticamente la que su madre manifiesta hacia él mismo. De esta forma, la perspectiva de segunda persona es condición de posibilidad no sólo de que el bebé se reconozca como agente, sino también de que adquiera la capacidad de distinguirse a sí mismo de los objetos de su entorno.

\section{Regulación afectiva y autoridad. La autoridad de segunda persona}

Mediante la atención conjunta he puesto de relieve la existencia de una capacidad preteórica del niño, la lectura de atención, mediante la cual el niño es capaz de interaccionar intencionalmente con un adulto sin poseer conceptos. Para dar un paso más allá y llegar a mostrar cómo dicha interacción puede llegar a ser normativa, es decir, cómo puede llegar a estar sujeta a criterios de corrección, debemos tener en cuenta, como ya hemos visto, un elemento clave: la emoción.

Aún cuando en la actualidad no tenemos una definición unánimemente aceptada de lo que es una emoción, las diversas posturas en teoría de la emoción admiten que las emociones tienen una función adaptativa, pues, al menos algunas, son dispositivos biológicos ${ }^{7}$ que nos ayudan a responder a las diversas situaciones a las que nos enfrentamos en la vida.

Los dispositivos biológicos de respuesta al entorno, innatos en todos los animales, regulan la actividad física, a partir de la percepción evaluadora (Pérez Jiménez 2009) ${ }^{8}$, para que podamos responder de la mejor forma posible a la situación en la que nos encontramos. Esto ocurre a veces de manera casi imperceptible, como cuando nuestras pupilas aumentan de

7 No todas las emociones pueden ser consideradas como dispositivos biológicos innatos. Algunas emociones son el resultado del desarrollo en un entorno social y surgen en una edad avanzada, cuando el niño ya se ha convertido en un ser conceptual propiamente dicho. Algunos ejemplos de este tipo de emociones son el resentimiento y la gratitud.

8 En este trabajo, Pérez realiza un exhaustivo análisis del desarrollo del niño desde sus primeros días de vida hasta que llega a convertirse en un ser conceptual propiamente dicho. Pérez ofrece una minuciosa y excelente explicación de este proceso basándose en lo que el llama el Principio de Caridad Emotiva. 
tamaño ante una persona que nos atrae, y otras veces de manera evidente, como cuando nos sonrojamos ante una situación bochornosa. De esta manera, las emociones comunican a los otros las valoraciones que hacemos mediante expresiones características externas.

La interacción emocional establece formas de comunicación entre dos sujetos emocionales y un entorno común a ambos, de una forma que regula su actividad recíproca. Podemos hablar, por tanto, de una regulación mutua del comportamiento basado en la percepción de las emociones ajenas, que en el caso de la interacción emocional entre un niño y su madre se torna en una forma de regulación afectiva que normativiza la conducta del niño.

Un ejemplo de este tipo de regulación afectiva entre niños de nueve meses en adelante y el adulto es la denominada 'asignación de referencia social' (Campos et al. 198, c.p. Hobson 1993, 57). El experimento (Sorce et al. 1985, c.p. Hobson 1993, 57) se desarrolla poniendo al bebé a gatear encima de una pasarela al final de la cual se coloca un objeto de su interés y en torno al cual se sitúa su madre, de forma que el bebé tenga acceso visual a ambos. El bebé comienza a gatear en dirección al objeto, pero en un momento determinado se encuentra con que hay un precipicio que lo separa del mismo. Lo que ocurre en realidad es que esa parte de la pasarela está construida con una superficie transparente, de manera que el niño sólo puede ver la gran altura que lo separa del suelo sin ser consciente de que está tapado con dicha superficie. En los experimentos realizados, muchos bebés no notaron el precipicio y continuaron gateando hacia el objeto. Asimismo, muchos otros sí lo notaron $\mathrm{y}$, consecuentemente, se detuvieron ante él. De estos últimos, un gran número levantó la cabeza en busca de la mirada de su madre. Los resultados de estos experimentos indican que, ante esta situación, el $75 \%$ de los bebés continúa su camino si la madre responde a la mirada del bebé con una expresión favorable, que comunica un valor positivo, como por ejemplo con un gesto de sonrisa y amabilidad. Por el contrario, si la madre responde con un gesto negativo, como una expresión de temor, ninguno de los bebés continua su camino. Cuando la madre pone una expresión de rabia sólo un $9 \%$ de los bebés continua mientras que alrededor de un $80 \%$ retrocede.

Es por ello que Hobson afirma que

Los bebés de alrededor de un año parecen ser capaces de sondear la expresión afectiva de su madre, relacionarla con una determinada situación y reaccionar sentimental y activamente en consecuencia. Los bebés parecen reconocer que la expresión de la otra persona posee un significado referido a un ambiente que comparten con ella. (Hobson 1993, 57) ${ }^{9}$.

Según esto, el vínculo psicológico que se crea entre la madre y el niño, a través de interacción emocional, es el que posibilita la apropiación psicológica del valor de los sucesos por parte del niño.

Cuando el bebé se enfrenta a una experiencia desconocida para él, para la que no tiene un valor asignado, es la expresión que capta en su madre la que le hace actuar en una u otra

9 En el apartado anterior hemos visto como el bebé de muy temprana edad ya es capaz de captar los estados afectivos de su madre y responder a ellos con sus propios estados afectivos, aquí vemos como a partir de los nueve meses el bebé es capaz de ponerlos en relación con una situación externa por ambos compartida. 
dirección. El bebé se encuentra ante tres elementos diferenciados: el suceso desconocido para él, la interacción con la madre, por medio de la mirada, y la respuesta emocional de ésta ante el elemento en cuestión. El acto de mirar hacia su madre, indica que el bebé busca en su expresión la asignación de un valor ante una situación que desconoce, con el objeto de usar dicho valor para orientar su conducta. La percepción emocional del bebé de la expresión emocional de la madre dirige, por tanto, la conducta del mismo, le indica qué debe hacer y qué no. En otras palabras, el bebé no posee una autoridad propia sobre el suceso y es la madre quien suple dicha autoridad.

La confianza emocional que el niño siente hacia su madre, y la autoridad que en un cierto sentido le otorga, permite que ésta regule su conducta en los momentos ocasionales en los que se da la interacción cara a cara. Por otra parte, la información valorativa que el niño obtiene de su madre es asimilada por éste para normativizar futuras conductas. El bebé aprende a través de la interacción cara a cara con su madre, a través de la autoridad que se origina en las ocasiones de triangulación afectiva, a actuar de manera similar en las ocasiones futuras en las que se encuentre en contextos semejantes.

Aun cuando para poseer una perspectiva de primera persona se requiere distinguir entre uno mismo y el entorno, es decir, que el niño sea capaz de sentirse como un agente diferenciado capaz de triangular emotivamente, todavía no es posible afirmar que dicha perspectiva surge en él durante este período, puesto que todavía no se trata de una perspectiva teórica que involucre conceptos ni conlleve autoridad.

Asimismo, como hemos visto al final de la sección anterior, siendo que el sentido de mundo externo del bebé surge cuando empieza a saber de sí en relación con su madre, podemos afirmar que el hecho de descubrirse como agente tiene como base a la perspectiva de segunda persona, es decir, a la interacción emocional cara a cara del bebé con su madre.

Sin embargo, aun cuando el niño ya es un agente consciente, es decir, un ser consciente de su capacidad de interaccionar con los otros y con el mundo externo, la autoridad que primeramente normativiza su conducta es la autoridad de su madre. Podemos, pues, afirmar tanto que el niño llega a descubrirse como agente gracias a la exposición a la perspectiva de segunda persona de la madre, como que adquiere una primera normatividad derivada de la autoridad de segunda persona de la misma.

\section{La perspectivas de primera y tercera persona y la autoridad de primera persona}

Resumiendo lo expuesto hasta este momento, hemos visto cómo a través de las interacciones con su madre surge en el niño la capacidad de descubrirse como agente, cómo dicha capacidad se amplia a los objetos del entorno apareciendo, a la edad aproximada de los nueve meses, la triangulación emocional a través de la cual y mediante la autoridad de segunda persona (mediante la asignaciones de valores no dependientes de él sino de las reacciones emotivas de su madre ante las situaciones que se le presentan cuando interactúa con ella), el niño aprende a normativizar su conducta, tanto ocasionalmente como a largo plazo. Ahora quisiera resaltar la importancia de la perspectiva de segunda persona en la aparición de la autoconciencia y, por ende, en la aparición de las perspectivas de primera y tercera persona.

El bebé percibe que su madre atiende a algo y ese algo es el propio bebé. De esta forma, incluso antes de ser plenamente conceptual, es decir, incluso antes de que se le 
pueda considerar un poseedor pleno de perspectivas, el bebé se descubre como objeto de atención de otro y, como consecuencia, descubre su capacidad para atender a objetos, mediante el seguimiento de la mirada del adulto. Aunque no quepa hablar de la existencia de perspectivas en el bebé hasta que éste sea un ser plenamente conceptual, tanto la capacidad de verse a sí mismo como objeto de la atención del adulto como la capacidad pre-reflexiva de atender a objetos son consecuencia de la exposición a una perspectiva de segunda persona hacia uno mismo.

Como he dicho, sólo cuando el niño es ya un ser plenamente conceptual, autoconsciente, se puede afirmar la aparición en él tanto de la perspectiva de primera persona como la perspectiva de tercera persona. Sin embargo, y ésta es la tesis central de este trabajo, ambas perspectivas descansan en la perspectiva de segunda persona. La perspectiva de primera tiene como condición necesaria, aunque no suficiente, reconocerse a sí mismo como agente a través del reconocimiento de sí mismo como objeto separado de la atención de la segunda persona. La perspectiva de tercera persona tiene como condición necesaria, aunque no suficiente, reconocer que no sólo él sino también otra persona (la segunda persona) puede atender a un objeto compartido por ambos. Esta última condición se manifiesta paradigmáticamente en la capacidad proto-declarativa que tiene el bebé de llamar la atención del adulto señalando objetos: tal capacidad implica ya la captación de la posibilidad de que lo señalado pueda ser objeto de atención de otra persona ${ }^{10}$.

Con la aparición de ambas perspectivas ya no es la madre exclusivamente quien regula el comportamiento del niño, sino que, junto con la perspectiva de primera persona, la adopción de la tercera persona sobre sí mismo, y su contraste con la de primera, posibilita que sea él quien corrija y modele su propio comportamiento. Podemos decir entonces que el niño ya posee una autoridad de primera persona, autoridad que tiene su base en la posibilidad de la adopción y contraste de ambas perspectivas, la suya propia, de primera persona, y la del otro, de tercera persona, aplicadas al entorno en el caso del conocimiento, o a sus propios estados mentales en el caso del autoconocimiento. Dicho de otra forma, la autoridad de primera persona queda constituida cuando el sujeto posee ya una perspectiva de primera persona y es capaz de interiorizar una perspectiva de tercera persona sobre sí mismo, siendo ambas perspectivas una consecuencia de estar expuesto, primero explícitamente y más tarde implícitamente, a la perspectiva de segunda persona ${ }^{11}$.

\section{Las perspectivas en la edad adulta}

Como he expuesto, es a través de la perspectiva de segunda persona como surgen tanto la perspectiva de primera como la de tercera persona, pues es mediante la perspectiva de segunda persona por la que el niño se descubre como agente, requisito imprescindible para

10 El señalamiento proto-declarativo emerge hacia el final del primer año de vida del bebé (9-12 meses), y se distingue de los señalamientos previos es que es intencionalmente dirigido (el bebé alterna la mirada entre el objeto y el adulto al tiempo que señala) y en que suele ir acompañado de otros comportamientos comunicativos como la vocalización. Franco (2005) sostiene que el señalamiento representa una herramienta única de gran alcance para del desarrollo de la comunicación y del lenguaje.

11 Un esbozo de la idea de que tanto la perspectiva de primera como la de tercera tienen como condición de posibilidad a la de segunda puede encontrarse en Pinedo (2004). 
la aparición de la perspectiva de primera persona, como lo es también para la aparición de la tercera el que el bebé reconozca que la otra persona puede atender al mismo objeto al que él está atendiendo, es decir, que su perspectiva no es la única, algo que tiene su origen en la interacción cara a cara con el adulto.

He mostrado también que la autoridad que aparece primeramente en el desarrollo ontogenético no es la de primera sino la de segunda persona (en el sentido laxo que he mostrado). Cuando aún no poseemos autoridad sobre nuestros estados mentales, es nuestra madre, a través de la triangulación emocional, quien tiene la capacidad de fijarlos ${ }^{12}$.

Siendo esto así, dicha triangulación, la capacidad de contrastar perspectivas, no tiene por qué quedar reducida al mero proceso de aprendizaje en la infancia.

Si esta explicación es válida, debe ir más allá de la infancia. Incluso en la edad adulta el involucramiento de este tipo con los objetos de atención de los demás continúa expandiendo nuestra conciencia de a qué tipo de cosas se les puede prestar atención y qué tipo de atención vale la pena prestarles. En muy probable que en diferentes relaciones y diferentes contextos culturales diferentes aspectos de los objetos y los tópicos se confirmen, subrayen u oscurezcan como focos de atención. (Reddy 2008, 117)

De esta manera, sostengo que tanto la perspectiva de primera, como las de segunda y tercera nos acompañan a lo largo de toda nuestra vida.

En «La perspectiva de segunda persona de la atribución mental» (Gomila 2002), Antoni Gomila hace una excelente descripción de la diferencia entre las tres perspectivas y de la particularidad de la perspectiva de segunda persona: En la edad adulta podemos caracterizar la perspectiva de primera persona como aquella en la que somos conscientes y autoconscientes, la de tercera persona como aquella en la que nos distanciamos en aras de la objetividad, y la de segunda persona como la del 'cara a cara', es decir, la de la interacción de las personas en la situación en la que estamos teniendo la comunicación. Los estados mentales que captamos mediante esta última perspectiva son estados expresivos, conformaciones corporales cuyo exponente principal es el rostro. Sin embargo, no se trata de una serie de rasgos físicos tales como movimientos musculares, entonaciones, posiciones, etc., que captemos e interpretemos, sino que son captados instantáneamente como un todo significativo sin período de reflexión alguno. De esta manera, los estados mentales que atribuimos mediante la interacción 'cara a cara' son «constitutivamente corporales, o bien, a la inversa, las actitudes y configuraciones corporales son también mentales» (Gomila 2002, 134).

12 En el apartado 4 hemos ilustrado esto con un ejemplo de la referencia social. Otro ejemplo de ello podría ser el de un niño pequeño, de menos de dos años, que se cae al suelo: lo primero que el niño hace es mirar a su madre, y según sea la respuesta de ésta así será la suya, es decir, si la madre se asusta el niño se asustará también, si la madre se queda tranquila el niño se levantará como si nada hubiera pasado. El niño procesa una serie de rasgos que capta instantáneamente y lo mueven a la acción. Es decir, el niño posee una capacidad preteórica, una capacidad de implicación emocional que al no ser filtrada por la autoridad de primera persona, pues carece de ella, se torna en autoridad de segunda persona. 
La instantaneidad, característica de la captación de estados mentales en la interacción cara a cara, hace que ésta influya en nosotros de manera directa, no pudiéndonos sustraer a dicha captación e influencia por ser éstas tanto emocionalmente constitutivas, como normalmente involuntarias y, por tanto, no conscientes.

$\mathrm{Si}$, como he sostenido anteriormente, la objetividad del conocimiento requiere de la posibilidad de la triangulación entre la perspectiva de primera persona, la de tercera y nuestros estados mentales, y nuestras interacciones con los demás se dan en segunda persona, debemos resaltar la importancia fundamental de esta última perspectiva, pues es a través de ella por la que las otras dos perspectivas tienen su origen y por la que continúan siendo modificadas a lo largo de nuestra existencia.

\section{Bibliografía}

BENSUSAN, H. y PINEDO, M. de (2007), «When my own beliefs are not first-personal enough», Theoria 58, 35-41.

BORGONI, C. (2009), Knowing the nature of one's mind, an externalist basis for selfknowledge. Tesis doctoral. Universidad de Granada.

BRAZELTON, T. B.; KOLOWSKI, B. y Main, M. (1974), «The origins of reciprocity: The early mother-infant interaction», en M. Lewis y L. Sosenblum (Eds.) The effect of the infant on its caregiver, Nueva York, Wiley, pp. 49-76.

BRETHERTON, I.; MCNEW, S., y BEEGHLY-SMITH, M. (1981), «Early person knowledge as expressed in gestural and verbal communication: When do infants acquire a 'theory of mind'?», en M. E. Lamb y L. R. Sherrod (Eds.), Infant social cognition: Empirical and theoretical considerations. Hillsdale, NJ, Lawrence Erlbaum Associates Inc, pp. 333-373. Brinck, I. (2004), «Joint Attention, Triangulation and Radical Interpretation: A Problem and its Solution», dialectica, 58 (2), 179-205.

CAMPOS, J. J. y STENBERG, C. R. (1981), «Percepcion, appraisal and emotion: The onset of social referencing», en M. E. Lamb y L. R. (Eds.), Infant social cognition: Empirical and theoretical considerations. Hillsdale, NJ, Lawrence Erlbaum Associates Inc., pp. 273-314.

COHN, J. F., y TRONICK, E. Z. (1983), «Three-month-old infants' reaction to simulated maternal depresión», Child Development, 54, 185-193.

DAVIDSON, D. (1982), «Animales racionales», en Subjetivo, intersubjetivo, objetivo. Trad.: Olga Fernández Prat. Madrid, Cátedra, Teorema, 2003, pp. 141-155.

- (1984), «La autoridad de primera persona», en Subjetivo, intersubjetivo, objetivo. Trad.: Olga Fernández Prat. Madrid, Cátedra, Teorema, 2003, pp. 25-40.

- (1991), «Tres variedades de conocimiento», en Subjetivo, intersubjetivo, objetivo. Trad.: Olga Fernández Prat. Madrid, Cátedra, Teorema, 2003, pp. 280-300.

DESCARTES, R. (1641), Meditaciones metafísicas. Trad. de Guillermo Graíno Ferrer. Madrid, Alianza, 2011.

EILAN, N. (2005), «Joint Attention, Communication and Mind», en Eilan, N.; Hoerl, Ch.; McCormack, T. Roessler, J. (Eds.), Joint Attention: Communication and Other Minds, Oxford, Oxford University Press., pp. 1-33. 
FARRONI, T., MANSFIELD, E. M., LAI, C, JOHNSON, M. H. (2003), «Infants perceiving and acting on the eyes: tests of an evolutionary hypothesis», Journal of Experimental Child Psychology, 85 (3), 199-212.

FRANCO, F. (2005), «Infant Pointing Harlequin, Servant of two masters», en Eilan, N.; Hoerl, Ch.; McCormack, T. Roessler, J. (Eds.), Joint Attention: Communication and Other Minds, Oxford, Oxford University Press.), pp. 129-164.

GOMILA, A. (2002), «La perspectiva de segunda persona de la atribución mental», Ediciones Universidad de Salamanca, Azafea. Rev. Filos. 4, 123-138.

HAVILAND J. M., y LELWIKA, M. (1987), «The induced affects responses: 10-week-old infant's reponses to three emotions expresions», Development Psycology, 23, 97-104.

HOBSON, P. (1993), El autismo y el desarrollo de la mente. Trad.: Angel Rivière Gómez. Madrid, Alianza Editorial, 1995.

MORAN, R. (2001), Authority and Estrangement - An Essay on Self-Knowledge, Princeton, Princeton University Press, 2001.

PÉREZ JIMÉNEZ, M.A. (2009), La aparición del pensamiento. Una enmienda emotiva a la tesis de Donald Davidson. Tesis doctoral. Universidad de Granada.

PINEDO, M. de (2004), «De la interpretación radical a la fusión de horizontes: la perspectiva de la segunda persona», en J. J. Acero et al. (Eds.), El legado de Gadamer, Granada, Universidad de Granada, pp. 225-235.

REDDY, V. (2008), How Infants Know Minds, Harvard, Harvard University Press.

RORTY, R. (1965), «Mind-Body Identity, Privacy, and Categories», Review of Metaphysics $19,24-54$.

RORTY, R. (1970), «Incorrigibility as the Mark of the Mental», Journal of Philosophy 67, 399-429.

RYLE, G. (1949), El concepto de lo mental. Trad.: E. Rabossi. Barcelona, Paidós, 2005.

SORCE, J. F., EMDE, R. N., CAMPOS, J., KLINNERT, M. D. (1985), «Maternal Emotional Signaling: Its Effect on the Visual Cliff Behavior of 1-year-olds», Developmental Psychology, 21, 195-200.

WOLFF, P. H. (1969), «The natural history of of crying and other vocalizations in early infancy», en B. M. Foss (Ed.), Determinants of infant behavior, Londres, Methuen, Vol. IV, pp. 81-109. 
